\title{
Automation of waste recycling using hyperspectral image analysis
}

\author{
Artzai Picon $^{1} \quad$ Ovidiu Ghita $^{2} \quad$ Pedro $^{\mathrm{a}}$ Iriondo $^{3} \quad$ Aranzazu Bereciartua $^{1}$ Paul F. Whelan ${ }^{2}$ \\ apicon@robotiker.es ghitao@eeng.dcu.ie pedro.iriondo@ehu.es abereciartua@robotiker.es paul.whelan@dcu.ie \\ ${ }^{1}$ Tecnalia Research and Innovation, Infotech Unit, Parque Tecnológico Ed 202 Robotiker-Tecnalia, \\ Zamudio, Spain. \\ ${ }^{2}$ Centre for Image Processing and Analysis, Dublin City University, Ireland. \\ ${ }^{3}$ Department of Automatic Control and Systems Engineering, University of the Basque Country, \\ Spain
}

\begin{abstract}
The advent of new hyperspectral imaging modalities made possible the implementation of flexible machine vision systems that can be designed to solve a variety of industrial tasks such as automatic material sorting. However the design of robust machine vision systems is far from a trivial task as several issues including mechanical design, development of an appropriate illumination set-up, optimal interfacing between the sensing and optical equipment with the computer vision component have to be properly addressed in order to accommodate all challenges that are encountered in a typical industrial environment. In this paper we present a novel methodology to automate the recycling process of non-ferrous metal Waste from Electric and Electronic Equipment (WEEE) where a particular emphasis is placed on the design choices that were made in the development of the proposed waste sorting system. The developed machine vision system has been subjected to a thorough robustness evaluation and the reported experimental results indicate that the proposed solution can be used to replace the manual procedure that is currently used in WEEE recycling plants.
\end{abstract}

\section{Introduction}

The last published reports indicate that the retired EEE constitutes $4 \%$ of the total municipal waste in Europe and is increasing by $16-28 \%$ every five years $[1,2]$. In this context, it is useful to note that the European Economic Area (EEA) countries generate 6.5 million tonnes of WEEE per annum and currently approximately $90 \%$ of this potentially hazardous waste is disposed as unsorted in generic municipal landfills. According to the current statistics, the EEE waste is growing 3 times faster than the standard municipal waste and the overall WEEE is expected to increase to 12 million tones by 2015 .

Although substantial efforts have been devoted to improve the WEEE recycling process [4], the success of current technologies available in recycle plants has been limited as the EEE scrap is formed by a relative large mix of different materials. The methods that are currently used to break the electronic equipment in their constituent parts require a labour intensive procedure, a fact that makes the recycling process not only time consuming but also very expensive. In the standard recycling process only the largest Aluminium, Copper and Stainless Steel parts are separated, since the cost and the time requirements to manually sort the rest of the shredded EEE waste are too prohibitive. To provide some insight into the cost of the recycling process, the financial demand to recycle cars or washing machines is approximately 100 Euro per tonne, whereas the estimated cost to recycle a tonne of electronic equipment is six times larger. However, besides processing costs (which are crucially important in any efficient industrial activity), it is useful to note that often the EEE waste has associated a high level of toxicity, a fact that projects a completely different perspective on the overall value of the WEEE recycling and disposal processes. As indicated earlier, it is estimated that more than 6 million tonnes of electrical equipment will be retired in the EEA countries this year, and in addition to issues related to the high level of toxicity and the substantial costs required to maintain and extend the municipal landfills where WEEE is disposed, it is useful to mention that the improper recycling of EEE precludes the further use of valuable materials as follows,

- 2.4 million tonnes of ferrous metal

- 1.2 million tonnes of plastic

- 652,000 tonnes of Copper

- 336,000 tonnes of Aluminium

- 336,000 tonnes of glass

Due to the aforementioned factors, legislation that sets specific requirements and targets with the aim of reducing WEEE quantity and its negative impact on the environment has been recently introduced. In this regard, the introduction of the European Directives 2002/96/EC on WEEE [3] and 2000/53/CE on End-of-Life-Vehicle has generated a completely different approach towards 
EEE recycling. This legislation will force companies to adjust their environmental policies, where product recycling being a prime example. As a result, new technologies for WEEE recycling need to be developed and this generates a favourable scenario for small and medium enterprises (SMEs) to be involved in a business that is now viable from an economic perspective.

In particular the SMEs involved in the WEEE recycling process are interested in developing new technologies that allow the identification and separation of small sized $(10-50 \mathrm{~mm})$ non-ferrous fractions. This is motivated by the fact that the vast majority of the retired electronic equipment is shredded before it is subjected to recycling procedures. Therefore, after the application of technological processes including magnetic, mechanical and densitometric sorting, the resulting EEE scrap still contains significant quantities of non-ferrous metals (e.g. Aluminium, Copper, Zinc, Brass, and Lead) that cannot be sorted using standard procedures. This issue has negative economic implications since the value of recycled materials depends largely on purity, and the unsorted non-ferrous material fractions are sold at a much lower price. Hence, substantial research on new methodologies that are able to robustly sort non-ferrous materials is currently under development with the aim of increasing the added value of the recycling process. In this regard, the major objective of this paper is to detail the technology behind the development of a novel machine vision system that has been designed to robustly identify and sort non-ferrous materials in the hyperspectral domain.

\section{System architecture}

In this section the design choices that were made during the development phase of the proposed material sorting system will be analysed and the main emphasis will be placed on issues related to material distribution, illumination set-up, hyperspectral image acquisition, non-ferrous material classification and material sorting.

In order to devise a flexible machine vision solution we have adopted a modular approach. The proposed system consists of three main components and a block diagram that shows the logic arrangement of the constituent modules is depicted in Figure 1. The first component of the proposed system has the role to uniformly place the non-ferrous WEEE particles on the conveyor belt via a vibratory feeding system. The next module performs the hyperspectral image acquisition that is triggered by the arrival of the scrap at the inspection line and its role is to capture the image data that is used in the subsequent material classification process. The final module of the system performs material sorting based on the information provided by the classification process via a pneumatic part extractor. A detailed discussion about each component of the developed conveyor-based material sorting system will be provided in the next sub-sections of the paper.

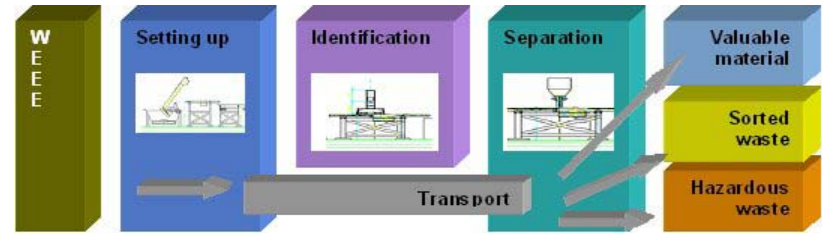

Figure 1. Overview of the designed material sorting system.

\subsection{WEEE vibratory feeder module}

The vibratory feeder module is an important part of the system and it has the role to place the non-ferrous particles in a uniform layer on the conveyor belt of the system. This module has been carefully designed to allow the particles to be arranged on the conveyor belt without overlaps and to offer a facile loading of the unsorted EEE scrap that has to be carried out by an operator. The component that places the WEEE particles on the conveyor belt consists of a group of mechanical elements that are activated in a sequential manner to implement a non-overlapping parts delivery system. The feeder module has been designed to ensure that the WEEE scrap mixture is placed in a thin layer to allow the implementation of an accurate and computationally efficient material classification process.

\subsection{WEEE particle transport system}

Once the particles that define the WEEE mixture are placed on the conveyor belt, they are transported to the inspection line to be classified and later sorted. In this process the speed of the belt is controlled by digital encoders to ensure a uniform image acquisition process and to preserve the information related to the position of the particles on the belt when they are sorted in the bins allocated for each material. The speed of the conveyor has been set in conjunction with the size of the nonferrous particles and the scan frequency of the hyperspectral image acquisition equipment. Based on these requirements the conveyor can be operated at speeds in the range 10 to $15 \mathrm{~m} / \mathrm{min}$, a performance that is compliant with the operational speed of the scrap transport systems that are used in recycling plants.

\subsection{Non-ferrous material identification module}

The non-ferrous material identification is the most sophisticated module of the system and it has three major sub-components, namely the illumination set-up, hyperspectral image acquisition and the material classification process. The illumination set-up is one of the key elements that have a significant impact on the overall performance of any machine vision system [6]. In the proposed implementation, the illumination module has been specifically designed to reduce the specular 
reflections generated by the surface of the non-ferrous materials and to provide a homogeneous and even illumination that covers the wavelengths that can be captured by the hyperspectral camera. Based on these premises, the illumination system consists of a parabolic surface that uniformly distributes the light generated by 9 halogens and 18 white LEDs covering the spectral range between 400 to $1000 \mathrm{~nm}$.

The second component of the non-ferrous material identification module involves the acquisition of the hyperspectral data that capture the characteristics of the non-ferrous materials that arrive at the inspection line. This technology involves the acquisition and interpretation of multi-dimensional digital images and the current range of spectral imaging systems is able to capture multiple bands from ultraviolet to far infrared with good bandwidth resolution. In our implementation we have employed a hyperspectral PHF Fast10 camera [5] that is able to capture wavelengths in the range 400 to $1000 \mathrm{~nm}$ with a spectral resolution of less than $1 \mathrm{~nm}$. The PHF Fast10 camera is fitted with a CMOS sensor (1024 × 1024 resolution), a Camera Link interface and a special Fore objective OL10. The hyperspectral camera is interfaced to an industrial PC computer using a standard National Instruments framegrabber.

The last component of the non-ferrous material identification module deals with the detection and classification of the non-ferrous particles using an analysis in the hyperspectral domain. The hyperspectral data can be thought of as a multi-dimensional image in which each layer corresponds to a certain wavelength (see Figure 2). Thus, each image pixel is defined by a vector where each component samples the amplitude of the optical signal for a particular wavelength.

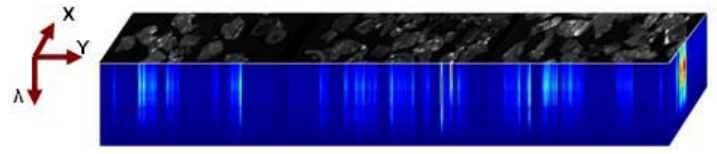

Figure 2. Hyperspectral image data.

While the hyperspectral data allow a more elaborate analysis of the properties associated with the non-ferrous materials than the standard RGB information, the main problem is the sheer amount of information that has to be evaluated during the classification process. To circumvent the computational related problems, decorrelation techniques are often applied to reduce the dimensionality of the hyperspectral data. In a recent paper [8] we have reported a novel classification algorithm that is based on the integration of spatial and spectral features in conjunction with a custom designed hyperspectral data decorrelation scheme.

The devised non-ferrous material classification algorithm entails two stages, a primary classification that is followed by a statistical region merging procedure. In the first step, the local histograms that sample the spatial-spectral features are calculated for each pixel in the image and they are compared with a priori models that are computed for each material during the training stage. In the second step a region merging reclassification is initiated to merge the regions that were incorrectly classified during the first stage due to problems caused by strong highlights and various oxidization levels. Full details about the proposed material classification can be found in [8].

\subsection{Material sorting module}

The material sorting module consists of 40 air blowing electrovalves and a PLC unit that controls their operation. This PLC unit also controls the vibratory feeder module and the speed of the conveyor belt using standard digital and analogic IO interfaces. The PLC unit communicates with the industrial PC and the information obtained after the application of the material classification process is used to estimate the position for each identified non-ferrous particle. Based on this information the PLC unit activates the adequate expulsion pneumatic valve to move the particles in the bins that are provided for each non-ferrous material.

\section{Experimental Results}

The system has been designed to perform the automatic identification and sorting of the following materials: white copper, aluminium, Stainless Steel, brass, copper and lead (see Figure 3).

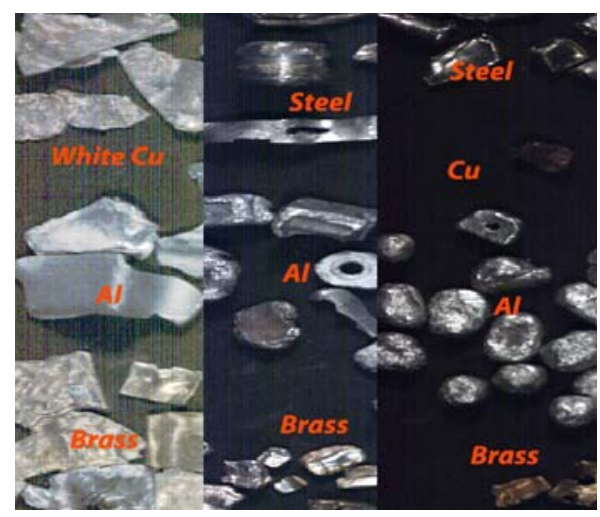

Figure 3. The non-ferrous materials investigated in this study.

The material samples that were used to validate the proposed material sorting system have been provided by Indumetal Recycling S.A. and IGE Hennemann Recycling $\mathrm{GmbH}$ which are part of the SORMEN project consortium [1]. The non-ferrous materials have been manually sorted by expert operators and in this process they used all available knowledge about each non-ferrous waste fraction. In this study the captured datasets were divided into training and testing sets where half of the data was used for training and the remaining half was used for testing. 
After the background pixels are identified using a pixel-based classification procedure, the hyperspectral data is subjected to data decorrelation to reduce its dimensionality. In our evaluation we have analysed the performance of several decorrelation schemes including Principal Component Analysis (PCA), Linear Discriminant Analysis (LDA), Wavelet decomposition, automatic band selection [7] and a new approach based on spectrum fuzzyfication [8]. The experimental results are depicted in Table I. In the first row of Table I the classification results using single-pixel spectral features are reported. These experiments have been conducted to identify the decorrelation scheme that returns the best performance. The results reported in Table I reveal that the decorrelation technique based on spectrum fuzzyfication outperformed the classical data decorrelation methods such as PCA, LDA, Wavelet and automatic band selection [8].

The experimental results shown in the second row were obtained when the spectral-spatial features were employed as features in the classification process. As expected, the experimental data clearly show that the fusion of spatial and spectral features is opportune as it samples in a more elaborate fashion the characteristics of the non-ferrous materials. The results depicted in the second row of Table I also indicate that the decorrelation technique based on spectrum fuzzyfication produces more consistent results than standard data decorrelation methods.

TABLE I

COMPARATIVE RESUlts: SINGLE-PIXEL DESCRIPTORS, FUZZY HISTOGRAMS AND REGION MERGING

\begin{tabular}{|c|c|c|c|c|}
\hline Algorithm & $\begin{array}{c}\text { PCA } \\
8 \text { features }\end{array}$ & $\begin{array}{l}\text { Fuzzysets } \\
8 \text { features }\end{array}$ & $\begin{array}{c}\text { Wavelet } \\
8 \text { features }\end{array}$ & $\begin{array}{c}\text { FSM } \\
8 \text { bands }\end{array}$ \\
\hline Single pixel & $66.43 \%$ & $71.52 \%$ & $63.79 \%$ & $62.03 \%$ \\
\hline Fuzzy histograms & $78.85 \%$ & $85.62 \%$ & $76.53 \%$ & $79.14 \%$ \\
\hline Region merging & $94.44 \%$ & $98.36 \%$ & $96.02 \%$ & $93.81 \%$ \\
\hline
\end{tabular}

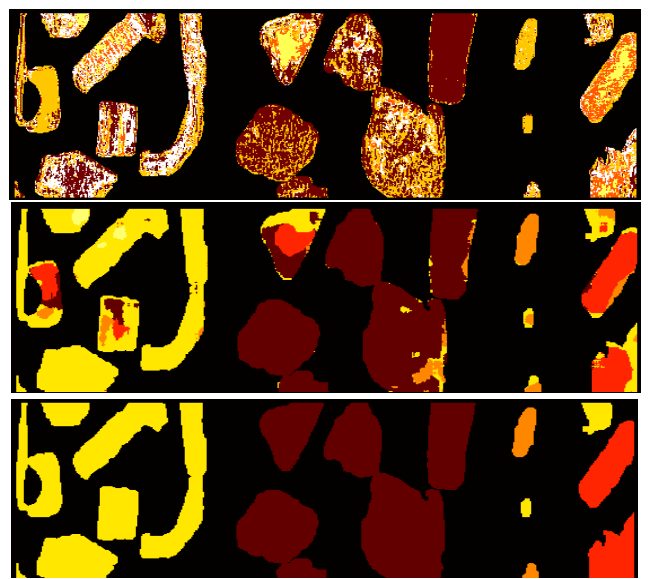

Figure 4. Classification results. (Top) Pixel-based classification results. (Middle) Classification results using spectral-spatial feature vectors. (Bottom) Classification results after region merging and re-classification.
As indicated in Section 2.3 the regions identified in the first step of the classification process (see the results in the second row of Table I) are subjected to reclassification using a statistical region merging approach [8] and the experimental results are shown in the third row of Table I. These results clearly indicate that the application of the region merging process reduced to a great extent the classification errors generated in the first step of the classification process. This is illustrated in Figure 4 where are depicted the results when the classification process has been carried out using singlepixel spectral features, spatial-spectral features and after the application of the statistical region merging process.

\section{Conclusions}

The aim of this paper was to detail the implementation of a novel machine vision system that addresses the automatic sorting of the EEE waste based on the evaluation of the properties associated with nonferrous materials in the hyperspectral domain. In this paper we have provided a comprehensive discussion about each module of the system and we have analysed a large spectrum of design choices that were made during the development stage of the proposed material sorting system. The reported experimental results show that the proposed non-ferrous material classification algorithm attained over $98 \%$ correct classification when applied to the identification of the WEEE scraps containing six different non-ferrous materials. This level of performance indicates that our system is a viable and cost-effective solution to the manual sorting procedure that is currently used in WEEE recycling plants.

\section{References}

[1] SORMEN - Innovative Separation Method for Non Ferrous Metal Waste from Electric and Electronic Equipment (WEEE) based on Multi- and Hyperspectral Identification project, Sixth Framework Programme Horizontal Research Activities Involving SMES CoOperative Research, 2006, http://www.sormen.org/

[2] A. Bereciartua, and J. Echazarra, "Sistema basado en identificación multiespectral para la separación de metales no férricos en WEEE en logísitica inversa", ler Congreso de Logística y Gestión de la Cadena de Suministro, 2007.

[3] Directive 2002/96/EC of the European Parliament and of the Council of 27 January 2003 on waste electrical and electronic equipment (WEEE) - Joint declaration of the European Parliament, the Council and the Commission relating to Article 9.

[4] D.B. Spencer, "The high-speed identification and sorting of nonferrous scrap", JOM Journal of the Minerals, Metals and Materials Society, vol. 57, no. 4, pp. 46-51, 2005.

[5] Specim Spectral Imaging Ltd. http://www.specim.fi/.

[6] B.G. Batchelor and P.F. Whelan (1997), Intelligent Vision Systems for Industry, Springer-Verlag (London), 457 pages, ISBN 3-540-19969-1.

[7] H. Grahn and P. Geladi (Eds.), Techniques and Applications of Hyperspectral Image Analysis, Wiley, ISBN-10: 0-470-01086-X, 2007.

[8] A. Picon, O. Ghita, P.F. Whelan, and P.M. Iriondo, "Fuzzy Spectral and Spatial Feature Integration for Classification of Nonferrous Materials in Hyperspectral Data", IEEE Transactions on Industrial Informatics, vol. 5, no. 4, pp. 483-494, Nov. 2009. 